\title{
Holistic Nature of Surya Namaskar for the Millennials, Reviewing and Investigating its Scientific Rationale
}

\author{
Mayank Shukla* \\ Amity Institute of Physiotherapy, Amity University, India \\ Submission: June 19, 2019; Published: July 19, 2019 \\ *Corresponding author: Mayank Shukla, Amity Institute of Physiotherapy, Amity University, India
}

\begin{abstract}
Background and Introduction: Surya Namaskar is a popular yogic practice. It is mainly an outdoor activity done in front of the rising sun. It has many beneficial promotive health effects. In the present-day outdoor activity and sun exposure is limited; Surya Namaskar may mitigate the ill effects of modern lifestyle.

Material and Methods: Data bases of DOAJ, PubMed and Google Scholar were searched with a focused research question, "what is the rationale of Surya Namaskar based yoga practice for holistic promotive health for futuristic generations"? 16 Full texts and 18 abstracts were extracted for review purposes.

Results and Conclusion: Surya Namaskar is a holistic exercise that gives daily dose of Vitamin D, flexibility training to ligaments and muscles, cognitive boost, cardiopulmonary tuning, spinal adjustments, bone health, glucose metabolism and psychological well-being to all age groups and genders.

Keywords: Surya Namaskar; Yoga; Health; Sun
\end{abstract}

\section{Introduction}

Surya Namaskar is an age old traditional and sequential set of sun prayer postures designed for holistic and good human health and societal well-being [1]. The benefits are diverse over a number of physical health systems and include psychological security as well through aerobic conditioning under the sun [2]; it is a classical requirement for future generations [3]. Need of the study - in the present and futuristic time the outdoor activity levels have reduced drastically. Individuals who exercise also prefer the indoor environment in a gym. This may produce a sun deficient population. In this scenario the holistic nature of Surya Namaskar may be very beneficial and may give a dose dependent daily boost for health. Aim was to review publications on Surya Namaskar and to investigate the findings for its holistic nature as applicable to millennials. The review question was "what is the rationale of Surya Namaskar based yoga practice for holistic promotive health for futuristic generations"?

\section{Methods}

Databases Directory of Open Access Journals (DOAJ), PubMed and Google Scholar were used as search engines. Key words were Surya Namaskar and Yoga. 16 Full texts and 18 abstracts were extracted for review purposes based on their suitability for the research(review) question. Results \& Analysis - DOAJ provided
2 full texts review articles, PubMed provided 10 articles (1 full text and 9 abstracts were seen at PubMed with key terms "Surya Namaskar", "Yoga". Many articles described low energy requirements for Surya Namasakr. Google Scholar provided 2010 results of different nature and suitable a total of 36 articles based on research question were selected for review from the literature search.

\section{Discussion}

Surya Namaskar shall be performed facing the Sun and it is sequence of 12 poses repeated one after the other in succession and interposed with pauses for slow breathing with focus on Sun God. It may also include chanting of mantras which give additional benefits. Based on review of the selected articles following areas of health were seen as a prominent focus for health for futuristic humans. Surya Namaskar as a daily dose of vitamin D - vitamin D is a fat-soluble vitamin that is directly produced by skin. Daily sun exposure during various asanas can increase the levels of vitamin D synthesis [4-6] and can work as a daily supplement, the asans may be performed at a suitable environment preferably during warm ambient sunlight in the morning [4-7]. Flexibility training - Surya Namaskar has been shown to increase dynamic flexibility of the chest wall $[8,9]$. It involves backwards and forward bending in the second and 
third poses where hip flexors anterior trunk and hamstrings and upper back are addressed for flexibility, in the fourth and sixth pose gastrocnemius are addressed for flexibility. There is cobra pose in the concluding aspects which is also very good for this purpose $[1,8,10]$.

\section{Weight Reduction}

Many articles have given sound rationale for the applicability of the yoga mainly in the form of Surya Namaskar for reduction of Obesity, however it must be clinically determined which treatment approach is best for the given patient [11-13]. Cognitive boost Surya Namaskar is a cognitive booster. Randomized controlled trial (RCT) has been reported in which Surya Namaskar has been shown to increase selective attention among orphan boys. Many other qualitative studies have confirmed sound rationale for improving cognition and mindfulness with the practice of Surya Namaskar [14-21].During Surya Namaskar the focus is drawn internally and the thinking process is diverted towards the yogic exercise, there is coordinated movements with breathing control and sequential flexion and extension that helps to relieve stress, increase mindfulness and to develop a calm outlook.

\section{Cardiopulmonary Tuning}

the energy requirement of Surya Namaskar is low, nevertheless it can be used very effectively for cardiopulmonary tuning. In the forward bending postures, it causes cardiopulmonary adjustments and leads to tuning of the reflexes which modulate the blood pressure and breathing drive. Sun light produces relaxation of blood vessels with the increase in nitric oxide. Many studies including school children, medical students etc. has confirmed its efficacy for cardiopulmonary tuning [22-30].

\section{Glucose Metabolism}

Surya Namaskar increases the efficiency of body to metabolize glucose, the endocrinal boost usually comes with a better circulation and energy levels remain high [31-37]. Two reviews [32,33] and a randomized controlled trial has proven the effectiveness of yoga intervention for diabetes [37]. Cardiometabolic risk I also decreased by it [38]. Spinal adjustments A systematic review and meta-analysis a has recommended yoga for low back pain. The 12 exercises of the Surya Namaskar cover both extension and flexion postures for the spine and in case of asymptomatic individuals can be used as a promotive exercise intervention. In case of low back pain Surya Namaskar can lead to better spinal adjustments as it is a self-controlled spinal adjustment [39].

\section{Psychological well-being}

During menopause various symptoms can be eased by regular practice of yoga as checked by RCT [40]. Surya Namaskar based yoga can improve the bone health as well. It can also increase physical performance before puberty [41], thus all age groups can benefit with its practice.

\section{Conclusion}

based on the findings of this review it is concluded that Surya Namaskar is a holistic exercise that gives daily dose of Vitamin D, flexibility training, cognitive boost, cardiopulmonary tuning, spinal adjustments, glucose metabolism and psychological wellbeing.

\section{References}

1. Vaibhav A, Shukla S, Singh OP (2016) Surya Namaskar (Sun Salutation): A Path to Good Health. International Journal of Pharmacological Research 6(7): 224-230.

2. Hillman CH, Erickson KI, Kramer AF (2008) Be smart, exercise your heart: exercise effects on brain and cognition. Nat Rev Neurosci 9(1): 58.

3. Booth FW, Laye MJ (2010) The future: genes, physical activity and health. Acta physiologica 199(4): 549-556.

4. Sri Ravi Shankar, Yogic Sunbaths boosts vitamin D. Art of living foundation The Art of Living International Center 560082, India.

5. Lehertn P, Villaseca P, Hogervorst E, Maki PM, Henderson VW (2015) Individually modifiable risk factors to ameliorate cognitive aging: a systematic review and meta-analysis. Climacteric 18(5): 678-689.

6. Shukla M, Shenoy S, Sandhu JS (2012) Low Ultraviolet Index in Winter with Concomitant Hypovitaminosis D in Northern Indian Region (Amritsar) - A Pilot Study. International journal of life sciences 1(3): 64-67.

7. Chacko A (2013) Worship of Sun in RGGEDA. International Journal of Scientific and Research Publications 3(2).

8. Stec K, Choudhary R, Kulmatycki L (2010) The Effects of Dynamic Surya Namaskar on Differential Chest Circumference of Physical Education Students. Human Movementm 11(2): 179-183.

9. Deshmukh A, Chincholkar S, Sutar A, Ghodey S (2018) A comparative study between Pilates and Suryanamaskar on flexibility in women having sedentary lifestyle using sit and reach test and shoulder and wrist test International Journal of Yoga, Physiotherapy and Physical Education 3(2): 190-195.

10. Hong SY, Park J, Hah CK (2009) Comparison of Rom and muscle activities according to the skills of surya namaskara in vinyasa yoga. Korean Journal of Sport Biomechanics 19(1): 127-138.

11. Bhardwaj PR, Bhardwaj AK (2015) Therapeutic applications of yoga for weight reduction in obese population: an evidence-based overview. Online Journal of Multidisciplinary Research 1(1): 1-5.

12. Nautiyal R (2016) Effect of Surya Namaskar on weight loss in obese persons. Int. J. sci. and consciousness 2(1): 1-5.

13. Joshi DR, Adhikari N (2017) A review on management of obesity: Pharmacological versus non-pharmacological approach. World Journal of Pharmacy and Pharmaceutical Sciences 7(2): 106-112.

14. Telles S, Singh N, Bhardwaj AK, Kumar A, Balkrishna A (2013) Effect of yoga or physical exercise on physical, cognitive and emotional measures in children: a randomized controlled trial. Child Adolesc Psychiatry Ment Health 7(1): 37.

15. Javadekar P, Manjunath NK (2012) Effect of Surya namaskar on sustained attention in school children. J Yoga Phys Ther 2(110): 2.

16. Kondam AG, Nagadeepa W, Jagan N, Jyothinath K, Suresh M et al (2017) The effect of yoga in improved cognitive functions in medical students: A comparative study. Natl J Physiol Pharm Pharmacol 7(1): $38-42$. 
17. Chaya MS, Nagendra H, Selvam S, Kurpad A, Srinivasan K (2012) Effect of yoga on cognitive abilities in schoolchildren from a socioeconomically disadvantaged background: a randomized controlled study. J Altern Complement Med 18(12): 1161-1167.

18. Kyizom T, Singh S, Singh KP, Tandon OP, Kumar R (2010) Effect of pranayama \& yoga-asana on cognitive brain functions in type 2 diabetes-P3 event related evoked potential (ERP). Indian J Med Res 131(5): 636-640.

19. Nagendra H, Kumar V, Mukherjee S (2015) Cognitive behavior evaluation based on physiological parameters among young healthy subjects with yoga as intervention. Computational and mathematical methods in medicine 13 .

20. Devi TM, Ganpat TS, Kumar S, Ramarao NH (2015) Surya namaskara training for enhancing selective attention in orphan boys: A randomized control study. Saudi Journal of Sports Medicine 15(1): 37.

21. Deorari M, Agarwal M, \& Shukla PA Study on the Effect of Surya Namaskar on Emotional Maturity and Psychological Well-being. Int J Yoga Allied Sci 1(2): 119.

22. Bhutkar PM, Bhutkar MV, Taware GB, Doijad V, Doddamani BR (2008) Effect of suryanamaskar practice on cardio-respiratory fitness parameters: A pilot study. Al Ameen J Med Sci 1(2): 126-129.

23. Kumar S (2011) Effects of Suryanamaskar on cardiovascular and respiratory parameters in school students. Recent Research in Science and Technology 3(10): 19-24.

24. Sinha B, Sinha TD (2014) Effect of 11 months of yoga training on cardiorespiratory responses during the actual practice of Surya Namaskar. International journal of yoga 7(1): 72-75.

25. Kondam A, Chandrasekhar M, Punita P, Varadharaju B, Suresh M et al. (2015) Combined effects of pranayama and suryanamaskar on dynamic spirometric values in normal young subjects. National Journal of Physiology, Pharmacy and Pharmacology 5(2): 79.

26. Sinha B, Sinha TD, Patha A, Tomer OS (2014) Effects of yoga training on blood pressure response during surya namaskar following eleven months of yoga practice in army men and yoga-trained individuals. International Journal of Clinical and Experimental Physiology 1(1): 51-56.

27. Ghorpade-Aher J, Patil A, Deshpande Y, Gaopande S, Phatak E (2017) A proposed framework for prediction of pulse, based on the effect of surya namaskar on different prakruti at different prahars of the day. In 2017 International Conference on Big Data Analytics and Computational Intelligence (ICBDAC) p: 11-16.

28. Shimpi A, Shetye J, Mehta A (2014) Comparison between effect of equal intensity training with Suryanamaskar or Physical Education activity or combination of both on Physical fitness in Adolescent Urban School children-A Randomized Control Trial: A Hypothesis. Journal of Medical Thesis 2(2):16-20.

29. Maniazhagu D, Soundrya B, Revathi S (2012) Isolated and Combined Effects of Prānāyāma And Sūryanamaskāra On Muscular Endurance and CardioRespiratory Endruance. Yoga Mimamsa 44(3): 200-205.
30. Karthik PS, Chandrasekhar M, Ambareesha K, Nikhil C (2014) Effect of pranayama and suryanamaskar on pulmonary functions in medical students. J Clin Diagn Res. 8(12): BC04-BC06.

31. Malhotra V, Singh S, Tandon OP, Sharma SB (2005) The beneficial effect of yoga in diabetes. Nepal Medical College journal: NMCJ 7(2): 145-147.

32. Aljasir B, Bryson M, Al-shehri B (2010) Yoga practice for the management of type II diabetes mellitus in adults: a systematic review. Evidence-Based Complementary and Alternative Medicine 7(4): 399-408.

33. Pandey A, Tripathi P, Pandey R, Srivatava R, Goswami S (2011) Alternative therapies useful in the management of diabetes: A systematic review. Journal of pharmacy \& bioallied sciences 3(4): 504-512.

34. Mody BS (2011) Acute effects of Surya Namaskar on the cardiovascular \& metabolic system. J Bodyw Mov Ther 15(3): 343347.

35. Chimkode SM., Kumaran SD, Kanhere VV, Shivanna R (2015) Effect of yoga on blood glucose levels in patients with type 2 diabetes mellitus. J Clin Diagn Res 9(4): CC01-CC03.

36. Mooventhan A (2017) A narrative review on role of Yoga as an adjuvant in the management of risk factor, disease progression and the complications of type 2 diabetes mellitus. Diabetes Metab Syndr 11: S343-S346.

37. Keerthi GS, Pal P, Pal GK, Sahoo JP, Sridhar MG, Balachander J (2017) Effect of 12 weeks of yoga therapy on quality of life and Indian diabetes risk score in normotensive Indian young adult prediabetics and diabetics: randomized control trial. J Clin Diagn Res 11(9): CC10CC14.

38. Yadav R, Yadav RK, Khadgawat R, Pandey RM, Upadhyay AD et at.al (2019) Randomized Controlled Trial of A 12-Week Yoga-Based (Including Diet) Lifestyle vs. Dietary Intervention on CardioMetabolic Risk Factors and Continuous Risk Score in Indian Adults with Metabolic Syndrome. Behav Med 1-12.

39. Cramer H, Lauchen R, Haller H, Dobos G (2013) A systematic review and meta-analysis of yoga for low back pain. Clin J Pain 29(5): 450460 .

40. Chattha R, Raghuram N, Venkatram P, Hongasandra NR (2008) Treating the climacteric symptoms in Indian women with an integrated approach to yoga therapy: a randomized control study. Menopause 15(5): 862-870.

41. Dsouza C, Avadhany S (2014) Effects of yoga training and detraining on physical performance measures in prepubertal children--a randomized trial. Indian J Physiol Pharmacol 58(1): 61-68. 